\title{
Melatonin: Revisited Role as Vaccine Adjuvant During Outbreaks of COVID-19 Caused by the Delta Variant
}

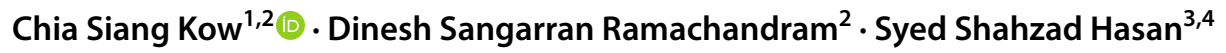

Received: 27 September 2021 / Accepted: 24 November 2021 / Published online: 30 November 2021

(c) The Author(s), under exclusive licence to Springer Science+Business Media, LLC, part of Springer Nature 2021

Dear editor,

We wish to reiterate the idea raised by Maestroni (2020) in which the administration of exogenous melatonin may boost the potency of immune response and duration of immunity conferred by coronavirus disease 2019 (COVID-19) vaccination. In fact, given the current circumstances where the emergence of variants of concern of severe acute respiratory syndrome coronavirus 2 (SARSCoV-2), especially the Delta variant (B.1.617.2), could significantly undermine the protective ability of currently available COVID-19 vaccines (Lopez Bernal et al. 2021; Sheikh et al. 2021), the suggestion to administer exogenous melatonin in order to boost further the immunity after COVID-19 vaccination becomes more important than before, and should be investigated in clinical studies.

There was a recent study (Jordan et al. 2021) which investigated $\mathrm{T}$ cell immunity in patients with documented SARSCoV-2 infection and individuals who had been fully vaccinated with BNT162b2 vaccine (1 month after the second vaccine dose) in order to identify $\mathrm{CD} 4+/ \mathrm{CD} 8+\mathrm{T}$ cell cytokine responses to SARS-CoV-2 and its variant peptides. As expected, T cells from SARS-CoV-2-infected or vaccinated individuals demonstrated substantial spikespecific CD $4+$ and $\mathrm{CD} 8+\mathrm{T}$ cell populations. Furthermore, the induced $\mathrm{T}$ cell reactivity to spike-specific peptides in vaccinated individuals was equivalent to that of infected

Chia Siang Kow

1 School of Postgraduate Studies, International Medical University, Kuala Lumpur, Malaysia

2 School of Pharmacy, Monash University Malaysia, Jalan Lagoon Selatan, Bandar Sunway, Subang Jaya, Selangor, Malaysia

3 School of Applied Sciences, University of Huddersfield, Huddersfield, United Kingdom

4 School of Biomedical Sciences \& Pharmacy, University of Newcastle, Callaghan, Australia patients after recovery. The researchers further investigated whether the delta variant evades $T$ cell immunity conferred by the BNT162b2 vaccine. To our amazement, there were nearly identical CD4 + and CD8 + T cell responses to the spike-specific peptides of Delta variant compared to spikespecific peptides of ancestral SARS-CoV-2. These findings suggest that the BNT162b2 vaccine could confer protective immunity against the Delta variant, but the key to this protective immunity lies in the memory $\mathrm{T}$ cells.

In fact, humoral immunity mediated by B cells dissipates overtime after COVID-19 vaccination. A multicenter, prospective cohort study (Favresse et al. 2021), which was designed to assess the antibody response in a population of healthcare professionals after the recipient of two doses of the BNT162b2 vaccine, reported significant antibody decline three months post-vaccination in both seronegative and seropositive individuals. Another prospective cohort study (Naaber et al. 2021) which investigated the antibody responses after the two-dose BNT162b2 vaccine in 122 volunteers observed that antibody levels at six months post-vaccination decreased on average to $7 \%$ of their peak level. Based on the findings, humoral immunity might not be reliable for long-term protection after COVID-19 vaccination, while eliciting T cell responses (cellular immunity) may be the key for COVID-19 vaccines to provide long-term protection against the Delta variant of SARS-CoV-2 (Noh et al. 2021).

As mentioned by Maestroni (2020), melatonin can enhance the immune response to a vaccine by increasing peripherally circulating CD $4+\mathrm{T}$ cells. Cited in the paper by Maestroni (2020) is an animal study (Ramos et al. 2008) which analyzed the effect of melatonin on T cell subsets in the peripheral blood of sheep vaccinated against $D$. nodosus and reported that administration of melatonin led to an increase in CD4 + T cell in vaccinated sheep. Another animal study (Baghban Rahimi et al. 2018) which evaluated the anti-tumor efficacy of the HPV-16 E7 DNA vaccine adjuvanted with melatonin, reported a significantly higher level of E7-specific $\mathrm{CD} 8+\mathrm{T}$-cell proliferation in the adjuvanted vaccine mice 
relative to non-adjuvanted vaccine mice. Although no human studies thus far evaluate the outcome of exogenous melatonin as a vaccine adjuvant, the effect of melatonin to enhance $T$ cell immunity has been well documented. For instance, a clinical trial (Lissoni et al. 1995) with AIDS patients reported that melatonin given orally at a dose of $40 \mathrm{mg} /$ day induced a significant increase in the number of $\mathrm{CD} 4+\mathrm{T}$ cells.

Protective immunity to SARS-CoV-2, especially of Delta variant, requires a more diverse immune response than antibodies alone. We believe exogenous melatonin administration could be used to harness the protective effects of memory T cells after COVID-19 vaccination to better protect against breakthrough infection. The literature gap of no human studies with melatonin as a vaccine adjuvant should be quickly addressed by performing more clinical research, especially in the current context of increasing uptake of COVID-19 vaccines. Finally, due to its immune-equilibrating action, melatonin might also be able to diminish the occurrence of adverse effects of COVID-19 vaccines, which may further boost the vaccine uptake.

Funding No funding was received to assist with the preparation of this manuscript.

Data Availability Statement Data sharing not applicable to this article as no datasets were generated or analysed during the current study.

\section{Declarations}

Conflict of Interest The authors have no relevant financial or non-financial interests to disclose.

\section{References}

Baghban Rahimi S, Mohebbi A, Vakilzadeh G, Biglari P, Jahromi SR, Mohebi SR, Shirian S, Gorji A, Ghaemi A (2018) Enhancement of therapeutic DNA vaccine potency by melatonin through inhibiting
VEGF expression and induction of antitumor immunity mediated by CD8 + T cells. Arch Virol 163(3):587-597

Favresse J, Bayart JL, Mullier F, Elsen M, Eucher C, Van Eeckhoudt S, Roy T, Wieers G, Laurent C, Dogné JM, Closset M, Douxfils J (2021) Antibody titres decline 3-month post-vaccination with BNT162b2. Emerg Microbes Infect 10(1):1495-1498

Jordan SC, Shin BH, Gadsden TM, Chu M, Petrosyan A, Le CN, Zabner R, Oft J, Pedraza I, Cheng S, Vo A, Ammerman N, Plummer J, Ge S, Froch M, Berg A, Toyoda M, Zhang R (2021) T cell immune responses to SARS-CoV-2 and variants of concern (Alpha and Delta) in infected and vaccinated individuals. Cell Mol Immunol 16:1-3

Lissoni P, Vigorè L, Rescaldani R, Rovelli F, Brivio F, Giani L, Barni S, Tancini G, Ardizzoia A, Viganò MG (1995) Neuroimmunotherapy with low-dose subcutaneous interleukin-2 plus melatonin in AIDS patients with CD4 cell number below 200/mm3: a biological phase-II study. J Biol Regul Homeost Agents 9(4):155-158

Lopez Bernal J, Andrews N, Gower C, Gallagher E, Simmons R, Thelwall S, Stowe J, Tessier E, Groves N, Dabrera G, Myers R, Campbell CNJ, Amirthalingam G, Edmunds M, Zambon M, Brown KE, Hopkins S, Chand M, Ramsay M (2021) Effectiveness of Covid-19 Vaccines against the B.1.617.2 (Delta) Variant. N Engl J Med 385(7):585-594

Maestroni G (2020) Exogenous melatonin as potential adjuvant in antiSarsCov2 vaccines. J Neuroimmune Pharmacol 15(4):572-573

Naaber P, Tserel L, Kangro K, Sepp E, Jürjenson V, Adamson A, Haljasmägi L, Rumm AP, Maruste R, Kärner J, Gerhold JM, Planken A, Ustav M, Kisand K, Peterson P (2021) Dynamics of antibody response to BNT162b2 vaccine after six months: a longitudinal prospective study. Lancet Reg Health Eur 6:100208

Noh JY, Jeong HW, Kim JH, Shin EC (2021) T cell-oriented strategies for controlling the COVID-19 pandemic. Nat Rev Immunol 8:1-2

Ramos A, Míguez MP, Morgado S, Sanchez-Correa B, Gordillo JJ, Casado JG, Tarazona R, Regodon S (2008) Melatonin enhances responsiveness to Dichelobacter nodosus vaccine in sheep and increases peripheral blood CD4 T lymphocytes and IgG-expressing B lymphocytes. Vet Immunol Immunopathol 206:1-8

Sheikh A, McMenamin J, Taylor B, Robertson C, Scotland PH, the EAVE II Collaborators, (2021) SARS-CoV-2 Delta VOC in Scotland: demographics, risk of hospital admission, and vaccine effectiveness. Lancet 397(10293):2461-2462

Publisher's Note Springer Nature remains neutral with regard to jurisdictional claims in published maps and institutional affiliations. 